\title{
Full Reconstruction of a Crystal Unit Cell Structure during Coherent Femtosecond Motion
}

\author{
S. L. Johnson, ${ }^{1, *}$ E. Vorobeva, ${ }^{1}$ P. Beaud, ${ }^{1}$ C. J. Milne, ${ }^{2}$ and G. Ingold ${ }^{1}$ \\ ${ }^{1}$ Swiss Light Source, Paul Scherrer Institut, 5232 Villigen PSI, Switzerland \\ ${ }^{2}$ Laboratoire de Spectroscopie Ultrarapide, Ecole Polytechnique Fédérale de Lausanne, 1015 Lausanne, Switzerland
}

(Received 10 July 2009; published 10 November 2009)

\begin{abstract}
We present a complete characterization of the unit cell dynamics of a laser-excited tellurium crystal using femtosecond $\mathrm{x}$-ray diffraction. The analysis offers a quantitative measure of the unit cell dynamics without making any assumptions on the symmetry of the excited-state motion. The results show a largeamplitude coherently excited $A_{1}$ mode quantitatively consistent with the predictions of a density functional theory model.
\end{abstract}

DOI: 10.1103/PhysRevLett.103.205501

PACS numbers: 63.20.K-, 61.80.Ba, 78.47.J-

The most fundamental time scale for structural dynamics in a crystal is set by the period of its vibrations. Along with the development of new sources of short subpicosecond pulses of $\mathrm{x}$ rays and electrons [1-5], there is a rapidly building interest in the development and use of diffraction-based techniques to experimentally characterize these vibrations and how they relate to the electronic states ultimately responsible for holding the crystal together [3,6-9]. In order to completely understand the dynamics of structure in response to a perturbation, the ideal diagnostic tool is the ability to construct a 3D "movie" of atom positions in the unit cell that allows us to draw conclusions without making a priori assumptions about the motion we expect to see and with enough time resolution to see the relevant dynamics. Femtosecond $\mathrm{x}$-ray diffraction offers the promise of exactly this kind of measurement.

The intensity of x-ray diffraction peaks is strongly related to the atomic structure of the unit cell via the structure factor

$$
F=\sum_{j} f_{j} e^{-i \mathbf{G} \cdot \mathbf{r}_{j}} e^{-W_{j}},
$$

where the index $j$ runs over all basis atoms, $f_{j}$ is the scattering form factor, $\mathbf{G}$ is a reciprocal lattice vector (equivalent to the momentum transfer from the crystal to the $\mathrm{x}$ rays), $\mathbf{r}_{j}$ is the average position of basis atom $j$ relative to a lattice point, and the Debye-Waller factor $e^{-W_{j}}$ represents the attenuation of the peak due to deviation of the basis atom $j$ throughout the crystal from its average position. In situations where kinematic theory is a good approximation, the scattered $\mathrm{x}$-ray intensity $I$ at a given diffraction peak follows the relation $I \sim|F|^{2}$. Conventional x-ray crystallography uses this to determine the average positions and variances of all atoms in the unit cell by careful measurement of a large number of peaks with different values of $\mathbf{G}$.

To achieve high time resolution with x-ray diffraction, one method is to employ a "pump-probe" scheme, where a crystal is repeatedly excited by an intense short optical pulse and the $\mathrm{x}$-ray diffraction is subsequently probed by a short x-ray pulse. By adjusting the relative delay of the two pulses, it becomes possible to track the structural changes in the crystal in response to optical excitation. A full unit cell characterization using such a technique has been demonstrated with 150 ps time resolution and $1.8 \AA$ spatial resolution for a myoglobin crystal, allowing us to watch how this protein functions as a $\mathrm{CO}$ ligand is swept away from its primary docking site after optical excitation [10]. In this Letter, we show a similar kind of structural reconstruction from $\mathrm{x}$-ray diffraction data for a much simpler crystal of tellurium, but with a considerably higher time resolution and spatial precision which allow us to see and characterize the motion of some of the fastest optical modes. This reconstruction does not rely on any assumptions about the symmetry of the coherent motion, and it allows us to test experimentally a preexisting model of how the bonding in this crystal is affected by the energy distribution of electrons.

For our measurements of the transient dynamics of a laser-excited crystal, we focus on a single crystal of tellurium. Under equilibrium conditions at room temperature, tellurium has space group $P 3_{1} 21$ (or the enantiomorphic group $P 3_{2} 21$ ) and a three atom basis arranged around a screw axis parallel to the $c$ axis of the hexagonal unit cell, forming a helical structure. Figure 1 shows a sketch of the unit cell, along with the six optical eigenmodes at zero wave vector. The average motion of the basis atoms within the unit cell causes changes in the x-ray intensity via $\mathbf{r}_{j}=$ $\mathbf{r}_{j}^{(0)}+\sum_{s} Q_{\mathbf{0} s} \boldsymbol{\epsilon}_{\mathbf{0} s}^{j}$, where $\mathbf{r}_{j}^{(0)}$ is the equilibrium position of atom $j, Q_{\mathbf{k} s}$ is the coordinate for the vibrational mode with wave vector $\mathbf{k}$ and branch index $s$, and $\boldsymbol{\epsilon}_{\mathbf{k} s}^{j}$ is the corresponding eigenvector for basis atom $j$. These eigenvectors form complete orthonormal sets [11] by the condition $\sum_{j}\left(\boldsymbol{\epsilon}_{\mathbf{k} s}^{j}\right)^{*} \cdot \boldsymbol{\epsilon}_{\mathbf{k} s^{\prime}}^{j}=\delta_{s s^{\prime}}$. If we approximate the atomic variance of the tellurium basis atoms as isotropic, we may write $W_{j}=G^{2}\left\langle u^{2}\right\rangle / 6$, where $G=|\mathbf{G}|$ and $\left\langle u^{2}\right\rangle$ is the variance of the atomic position. In thermal equilibrium, the variance is $\left\langle u^{2}\right\rangle_{\mathrm{eq}}=490 \pm 12 \mathrm{pm}^{2}$ at $293 \mathrm{~K}$ [12]. 


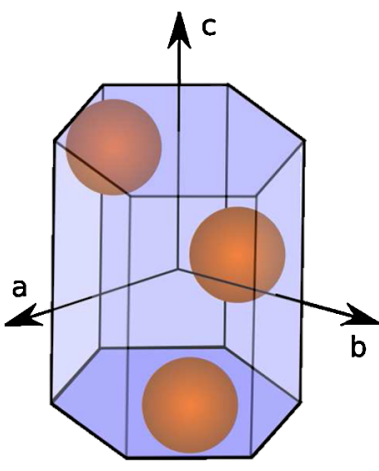

(a)

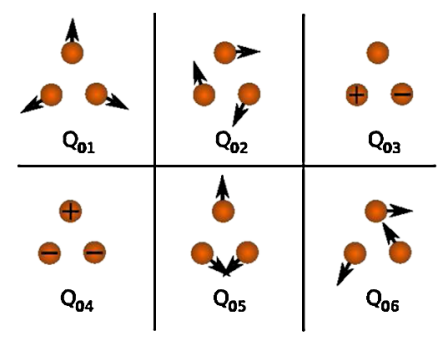

(b)
FIG. 1 (color online). Equilibrium unit cell structure and zero wave vector optical eigenmodes of tellurium. (a) The hexagonal unit cell of tellurium ( $a=4.456 \AA, c=5.921 \AA$ ), showing the three basis atoms arranged along a screw axis parallel to the $c$ axis. With this choice of origin, the basis atoms are located at $(x$, $0,0)\left(0, x, \frac{1}{3}\right)$, and $\left(-x,-x,-\frac{1}{3}\right)$, where $x=-0.2636$ at room temperature [12]. (b) The eigenmodes of the $\Gamma$-point optical modes in tellurium [13] viewed as a projection onto a plane normal to the $c$ axis of the unit cell.

Expanding Eq. (1) to first order in $Q_{0_{s}}$ and $\Delta\left\langle u^{2}\right\rangle=\left\langle u^{2}\right\rangle-$ $\left\langle u^{2}\right\rangle_{\text {eq }}$ gives

$$
\frac{I}{I_{\mathrm{eq}}}=1+2 \sum_{s} \operatorname{Im}\left\{\frac{\sum_{j}\left(\mathbf{G} \cdot \boldsymbol{\epsilon}_{\mathbf{0} s}^{j}\right) e^{-i \mathbf{G} \cdot \mathbf{r}_{j}^{(0)}}}{\sum_{j} e^{-i \mathbf{G} \cdot \mathbf{r}_{j}^{(0)}}}\right\} Q_{0 s}-\frac{1}{3} G^{2} \Delta\left\langle u^{2}\right\rangle,
$$

where $I_{\mathrm{eq}}$ is the equilibrium intensity of the diffraction peak. Measurements of the normalized diffracted intensity for several different values of $\mathbf{G}$ allow us to construct a system of linear equations that may be inverted to obtain values for the six optical mode coordinates $Q_{0 s}(s=$ $1 \ldots 6)$ and the change in atomic variance $\Delta\left\langle u^{2}\right\rangle$. A minimum of seven such reflections are required, provided the chosen peaks form a linearly independent set of equations. Measurement of additional reflections would allow greater precision and/or a relaxation of the assumption that the atomic variance is isotropic.

The femtosecond $\mathrm{x}$-ray diffraction from a single crystal of tellurium with a (011) surface was measured at an X-ray photon energy of $7.15 \mathrm{keV}$ using a synchrotron slicing source in a pump-probe grazing incidence geometry [4]. For all measurements, the angle of $\mathrm{x}$-ray incidence was kept fixed at $0.45^{\circ}$ to limit the field attenuation length of the $\mathrm{x}$ rays to $39 \mathrm{~nm}$ [14]. The crystal was excited by a femtosecond duration laser pulse $(800 \mathrm{~nm}, 115 \mathrm{fs}, 1 \mathrm{kHz})$ at a grazing incidence angle of $9.4^{\circ}$ with a polarization adjusted for each diffraction peak to maintain the orientation of the electric field of the pump pulse inside of the crystal perpendicular to the $c$ axis of the unit cell [15]. The energy of the pump pulse was also adjusted to keep the absorbed fluence constant at $1.2 \pm 0.2 \mathrm{~mJ} / \mathrm{cm}^{2}$. Total measurement time for each reflection varied depending on the diffraction efficiency but was typically around $8 \mathrm{~h}$.

Figure 2 shows the diffracted intensity as a function of pump-probe delay time for several diffraction peaks. For this geometry, the estimated time resolution of the experiment is 200 fs. By inverting Eq. (2) for these data, we obtain the normal mode coordinates and $\Delta\left\langle u^{2}\right\rangle$ as a function of pump-probe delay time, shown in Fig. 3. The $Q_{01}$ coordinate corresponds to the $A_{1}$ symmetry coherent mode previously inferred from optical reflectivity measurements [16-20]. In contrast to these optical measurements, our $\mathrm{x}$-ray diffraction data allow us to estimate directly the magnitude and direction of this motion. Here we clearly see a sudden increase in the quasiequilibium value of $Q_{01}$ by $1.2 \mathrm{pm}$, equivalent to an increase in the helix radius by $0.7 \mathrm{pm}$. This change drives a coherent phonon (vibrational mode) with frequency $3.44 \mathrm{THz}$. Hunsche et al. [18] have suggested that laser excitation causes a decrease in $Q_{01}$ based on a comparison of single-wavelength optical reflectivity data with the temperature dependence of the structure, a method first employed by Cheng et al. [21] to discuss the coherent dynamics of $\mathrm{Ti}_{2} \mathrm{O}_{3}$. This comparison may be misleading since it assumes a one-to-one correspondence between the nonequilibrium photoexcited state and the equilibrium state of the crystal at an elevated temperature. Density functional theory (DFT) calculations which consider the high electron-hole density created by intense photoexcitation [22] have predicted an increase in $Q_{01}$, a finding consistent with our direct measurement of the lattice dynamics.

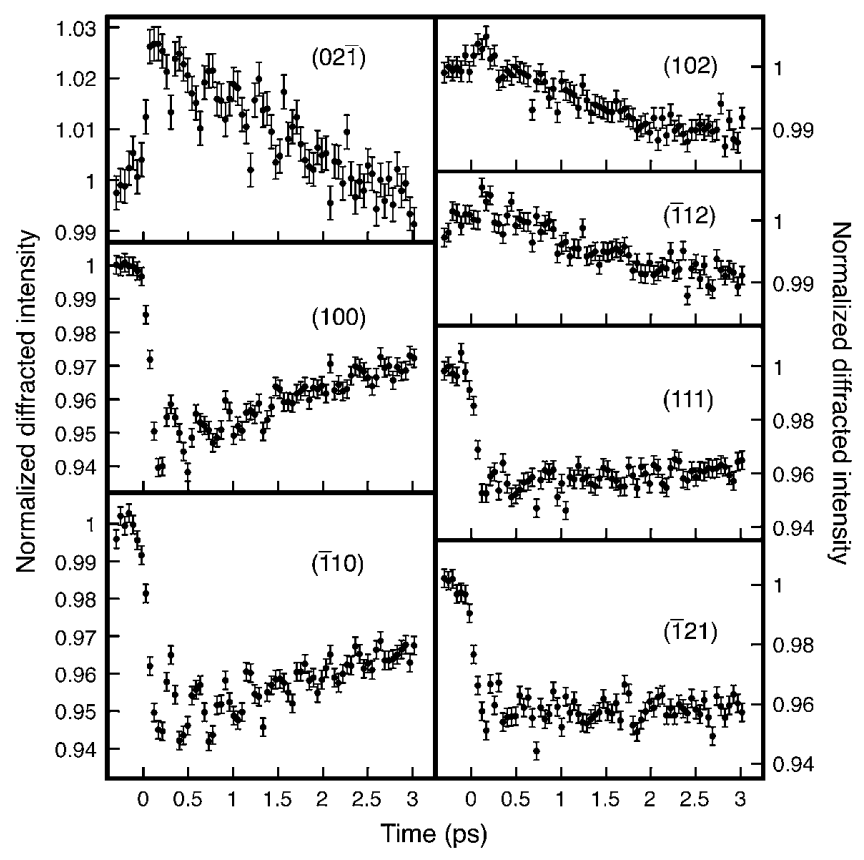

FIG. 2. Summary of femtosecond diffraction data from excited tellurium. All data were taken at a grazing incidence angle of $0.45^{\circ}$. The set of measured diffraction planes have been selected to form a linearly independent set that can be used to solve for the dynamics of the unit cell. 


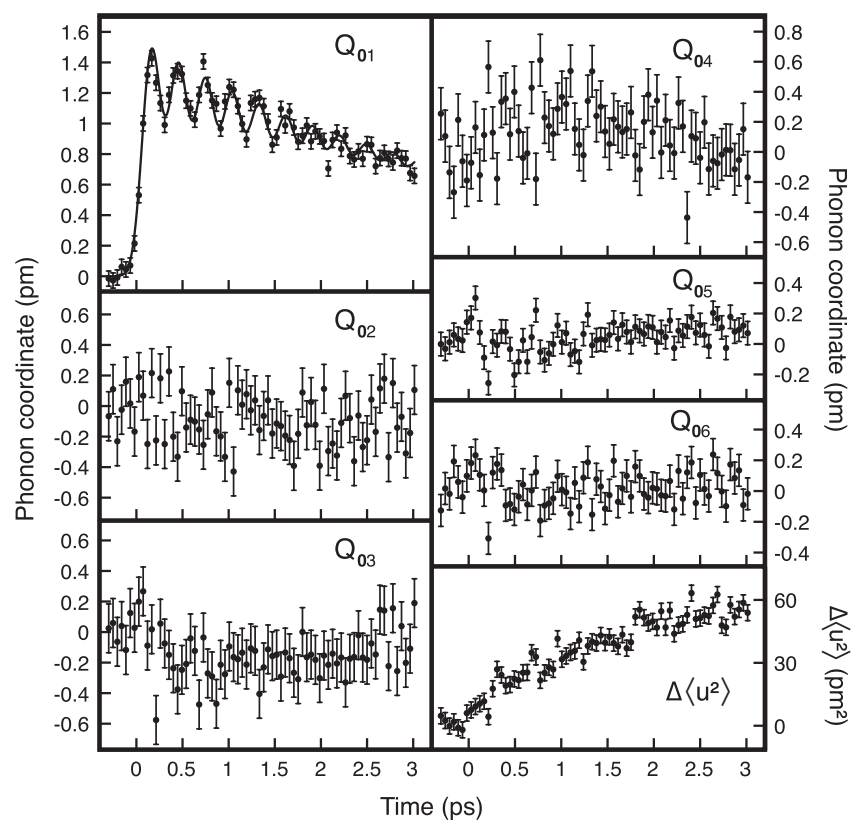

FIG. 3. Phonon mode and mean-square displacement dynamics of photoexcited tellurium. An inversion of the diffraction data from Fig. 2 allows a complete solution of the unit cell dynamics, equivalent to tracking the 3D position of each basis atom with $200 \mathrm{fs}$ time resolution. See the EPAPS supplement [23] for animated movies derived from this solution. The solid line superimposed over the $Q_{01}$ data shows the results of a fit to a displacive excitation model discussed in the text.

The solid line superimposed on the $Q_{01}$ data of Fig. 3 is a fit of the data to a simple model of displacive excitation for a fixed vibrational frequency and exponential damping constants for both the oscillation amplitude and the quasiequilibrium value of $Q_{01}$ after convolution with a Gaussian with full width at half maximum (FWHM) of 200 fs to account for the temporal resolution of the measurement [16]. This convolution reduces the magnitude of the coherent oscillations by a factor of 6 . The fitted frequency of $3.44 \pm 0.02 \mathrm{THz}$ compares well with the $3.42 \mathrm{THz}$ measured in optical reflectivity measurements by Hunsche et al. at nearly the same absorbed fluence [17]. The oscillation damping time is $1.4 \pm 0.2 \mathrm{ps}$, which is somewhat higher than the damping time of $1.05 \pm 0.2$ ps estimated from the Hunsche et al. optical measurements. One possible contribution to this damping time is the depth inhomogeneity of the probed region of the sample: as the distance from the surface increases, the excited carrier density decreases and causes the mode frequency to shift toward its equilibrium value of $3.6 \mathrm{THz}$. The longer damping time in the x-ray data may be because the $39 \mathrm{~nm}$ probe depth of the $x$ ray is slightly shorter than the $58 \mathrm{~nm}$ absorption depth of the optical probe [24]. To quantitatively estimate this effect and to separate it from other decay mechanisms (e.g., anharmonic decay) would require very accurate measurements at many different $\mathrm{X}$-ray incidence angles [25].
The observed magnitude of the $Q_{01}$ dynamics may be compared against the DFT calculations of photoexcited tellurium by Tangney and Fahy that assume the carrier density is a constant of motion in the crystal [22]. These calculations predict a relationship among the carrier density, the quasiequilibrium value of $Q_{01}$, and the frequency of the oscillations. For the first $1.5 \mathrm{ps}$ after excitation, when the oscillations are largest, our data show a quasiequilibrium shift of $1.15 \pm 0.15 \mathrm{pm}$. For this shift, the DFT predicts a frequency softening of $-0.19 \pm 0.02 \mathrm{THz}$ relative to the frequency in thermal equilibrium. This agrees with our measured frequency softening of $-0.16 \pm$ $0.03 \mathrm{THz}$. The calculations also predict that the excited carrier density under these conditions is $(2.9 \pm 0.3) \times$ $10^{20} \mathrm{~cm}^{-3}$. Although the carrier density is not measured in this experiment, we estimate from the absorbed fluence a carrier density of $(3.7 \pm 0.6) \times 10^{20} \mathrm{~cm}^{-3}$ at $1-2 \mathrm{ps}$ after excitation, averaged over the probe depth and taking into account losses from carrier diffusion [22,25].

The other vibrational coordinates depicted in Fig. 3 show no dynamics at levels beyond what might be ascribed to systematic errors arising from small differences in the absorbed pump fluence among the measured diffraction peaks (approximately equivalent to the magnitude of the error bars). Coherent dynamics of some of these modes have been observed in optical pump-probe measurements, excited by the transient electric fields created by the different diffusion rates of electrons and holes [26]. While these modes are probably excited to some extent in our experiment, we conclude that the amplitude of these vibrations is too small to detect from these data.

The changes in $\Delta\left\langle u^{2}\right\rangle$ shown in Fig. 3 show an increase in the atomic position variance over time, probably from a combination of excited-state softening of the acoustic mode frequencies [27] and incoherent energy transfer (heating) from photoexcited carriers. At 3 ps after excitation, the observed $\Delta\left\langle u^{2}\right\rangle \approx 55 \mathrm{pm}^{2}$ corresponds to an increase of $11 \%$ over the equilibrium value of $\left\langle u^{2}\right\rangle=$ $490 \mathrm{pm}^{2}$ estimated from static x-ray diffraction measurements [12]. As a rough qualitative guide, we can use a simple high-temperature Debye model that assumes thermal equilibrium within the lattice is maintained at all times and write $\left\langle u^{2}(t)\right\rangle=9 k_{B} T / 2 M \omega_{D}^{2}$, where $k_{B}$ is Boltzmann's constant, $T$ is the lattice temperature, $M$ is the atomic mass, and $\omega_{D}$ is a Debye frequency. In this model, the observed increase in the atomic position variance can be caused by an increase in $T$, a decrease in $\omega_{D}$, or a combination of the two. In the real crystal, the lattice temperature $T$ is not defined for times after excitation less than or comparable to a typical acoustic mode period, but this can in principle be generalized to a concept of an increase in the total energy of the phonon system without requiring a thermal population distribution. As previously shown in other materials $[7,25]$, both the frequency softening and incoherent energy transfer to the lattice are likely to contribute significantly to the observed increase in lattice disorder. 
We emphasize that the unique aspect of this experiment compared to previous studies is the ability to make a fully model-independent characterization of the dynamics of the Te unit cell. Measurement of the dynamics of only one or two diffraction peaks can offer a useful comparison to a preexisting model of the dynamics, but in the absence of such a model the dynamics cannot be understood unless there are sufficient data to make a full reconstruction of the unit cell. The $A_{1}$ symmetry expansion of the helix radius cannot be directly inferred from data from a subset of the reflections shown in Fig. 2, since it is not in principle possible to distinguish the $A_{1}$ motion from the contributions of other vibrational modes and the changes in the atomic variance. Since displacive excitation of other optical modes is known to occur [26], we require a full dynamical unit cell reconstruction to quantify the $A_{1}$ mode contribution and to place an upper bound on the excitation of the other eigenmodes of the unit cell.

Grazing incidence femtosecond $\mathrm{x}$-ray diffraction offers great potential to explore the complete dynamics of crystals on the fastest time scales relevant for structural motion. In the specific case of tellurium, these measurements appear to validate quantitatively the magnitude and character of lattice motion predicted from DFT models of the excited-state dynamics. More generally, this method allows us to experimentally map out the interatomic bond strength and structural dynamics of materials far from thermal equilibrium. In the near future, much more brilliant and short x-ray pulses produced by x-ray free electron lasers will allow the accurate measurement of the unit cell dynamics of more complex crystals, where precise measurements of $\sim 100$ distinct reflections are necessary but in principle possible with $10^{2}-10^{4}$ times greater flux compared to the current source. Such measurements can provide critical insight into the workings of the highly correlated systems that are currently a central focus of condensed matter physics and materials science [28].

These experiments were performed on the X05LA beam line at the Swiss Light Source, Paul Scherrer Institut, Villigen, Switzerland. We thank D. Grolimund and C. Borca for assistance during experiments.

*steve.johnson@psi.ch

[1] R. W. Schoenlein, S. Chattopadhyay, H. H. W. Chong, T. E. Glover, P. A. Heimann, C. V. Shank, A. A. Zholents, and M. S. Zolotorev, Science 287, 2237 (2000).

[2] A. Rousse, C. Rischel, and J.-C. Gauthier, Rev. Mod. Phys. 73, 17 (2001).

[3] A. M. Lindenberg et al., Science 308, 392 (2005).

[4] P. Beaud, S. L. Johnson, A. Streun, R. Abela, D. Abramsohn, D. Grolimund, F. S. Krasniqi, T. Schmidt, V. Schlott, and G. Ingold, Phys. Rev. Lett. 99, 174801 (2007).

[5] M. Chergui and A. H. Zewail, Chem. Phys. Chem. 10, 28 (2009).
[6] D. M. Fritz et al., Science 315, 633 (2007).

[7] R. Ernstorfer, M. Harb, C. T. Hebeisen, G. Sciaini, T. Dartigalongue, and R. J. D. Miller, Science 323, 1033 (2009).

[8] F. Carbone, P. Baum, P. Rudolf, and A. H. Zewail, Phys. Rev. Lett. 100, 035501 (2008).

[9] F. Carbone, D.-S. Yang, E. Giannini, and A. H. Zewail, Proc. Natl. Acad. Sci. U.S.A. 105, 20161 (2008).

[10] F. Schotte, M. Lim, T. A. Jackson, A. V. Smirnov, J. Soman, J. S. Olson, G. N. Phillips, M. Wulff, and P. A. Anfinrud, Science 300, 1944 (2003).

[11] Knowledge of the eigenvectors is convenient but not necessary for this analysis. They may be substituted by any complete orthonormal set of vectors (e.g., Cartesian basis vectors).

[12] C. Adenis, V. Langer, and O. Lindqvist, Acta Crystallogr. Sect. C 45, 941 (1989).

[13] P. Grosse, Die Festkörpereigenschaften von Tellur, Springer Tracts in Modern Physics Vol. 48 (SpringerVerlag, Berlin, 1969).

[14] The X-ray attenuation is dominated by the $39 \mathrm{~nm}$ photoabsorption depth; at the most efficient reflection studied, extinction effects cause this to decrease to a minimum of $34 \mathrm{~nm}$.

[15] The polarization of the pump field inside of the crystal was kept within $10^{\circ}$ of [210].

[16] H. J. Zeiger, J. Vidal, T. K. Cheng, E.P. Ippen, G. Dresselhaus, and M.S. Dresselhaus, Phys. Rev. B 45, 768 (1992).

[17] S. Hunsche, K. Wienecke, T. Dekorsy, and H. Kurz, Phys. Rev. Lett. 75, 1815 (1995).

[18] S. Hunsche, K. Wienecke, and H. Kurz, Appl. Phys. A 62, 499 (1996).

[19] A. M.-T. Kim, C. A. D. Roeser, and E. Mazur, Phys. Rev. B 68, 012301 (2003).

[20] S. I. Kudryashov, M. Kandyla, C.A. D. Roeser, and E. Mazur, Phys. Rev. B 75, 085207 (2007).

[21] T. K. Cheng, L. Acioli, J. Vidal, H. J. Zeiger, G. Dresselhaus, M. S. Dresselhaus, and E.P. Ippen, Appl. Phys. Lett. 62, 1901 (1993).

[22] P. Tangney and S. Fahy, Phys. Rev. B 65, 054302 (2002).

[23] See EPAPS Document No. E-PRLTAO-103-022947 for short animations depicting the results of the unit cell reconstruction discussed in the manuscript. For more information on EPAPS, see http://www.aip.org/pubservs/ epaps.html.

[24] S. Tutihasi, G. G. Roberts, R. C. Keezer, and R. E. Drews, Phys. Rev. 177, 1143 (1969).

[25] S. L. Johnson, P. Beaud, C. J. Milne, F. S. Krasniqi, E.S. Zijlstra, M. E. Garcia, M. Kaiser, D. Grolimund, R. Abela, and G. Ingold, Phys. Rev. Lett. 100, 155501 (2008).

[26] T. Dekorsy, H. Auer, H. J. Bakker, H. G. Roskos, and H. Kurz, Phys. Rev. B 53, 4005 (1996).

[27] S. L. Johnson, P. Beaud, E. Vorobeva, C. J. Milne, É. D. Murray, S. Fahy, and G. Ingold, Phys. Rev. Lett. 102, 175503 (2009).

[28] G. Ingold, R. Abela, P. Beaud, S. L. Johnson, and U. Staub, Z. Kristallogr. 223, 292 (2008). 